C9

the immunotherapeutic potential of oncolytic viruses
IMMUNOTHERAPY

\section{When viruses attack}

A limitation to the efficacy of immune checkpoint inhibitors is their dependence on a pre-existing antitumour immunity. Therefore, strategies to overcome tumour immunosuppression may improve the antitumour activity of current immunotherapies. Two studies now suggest this could be achieved with oncolytic virotherapy.

Ribas et al. report the results of a phase Ib clinical trial of a first-in-human combination immunotherapy that tested whether an oncolytic virus engineered to attract immune cells (talimogene laherparepvec) could improve the cytotoxic T lymphocyte (CTL) response to the anti-programmed cell death 1 (PD1) therapy pembrolizumab. Talimogene laherparepvec is a genetically modified form of herpes simplex virus type 1 optimized to replicate selectively in tumours and secrete granulocyte-macrophage colony-stimulating factor (GM-CSF), which increases antigen release, antigen presentation and systemic antitumour immunity. This virus was intratumourally injected into 21 patients with advanced melanoma, followed by combination dosing of the virus with intravenous pembrolizumab.

The combination therapy was generally well tolerated. A high objective response rate of $62 \%$ was observed and the complete response rate was $33 \%$. Of the patients who responded to the combination therapy, biopsies revealed that the initial single-agent injection of talimogene laherparepvec led to an increase in the levels of several infiltrating immune cell subsets in tumours, $\mathrm{CD}^{+} \mathrm{T}$ cells in particular, and elevated PD1 ligand 1 (PDL1) and interferon- $\gamma$ (IFNG)

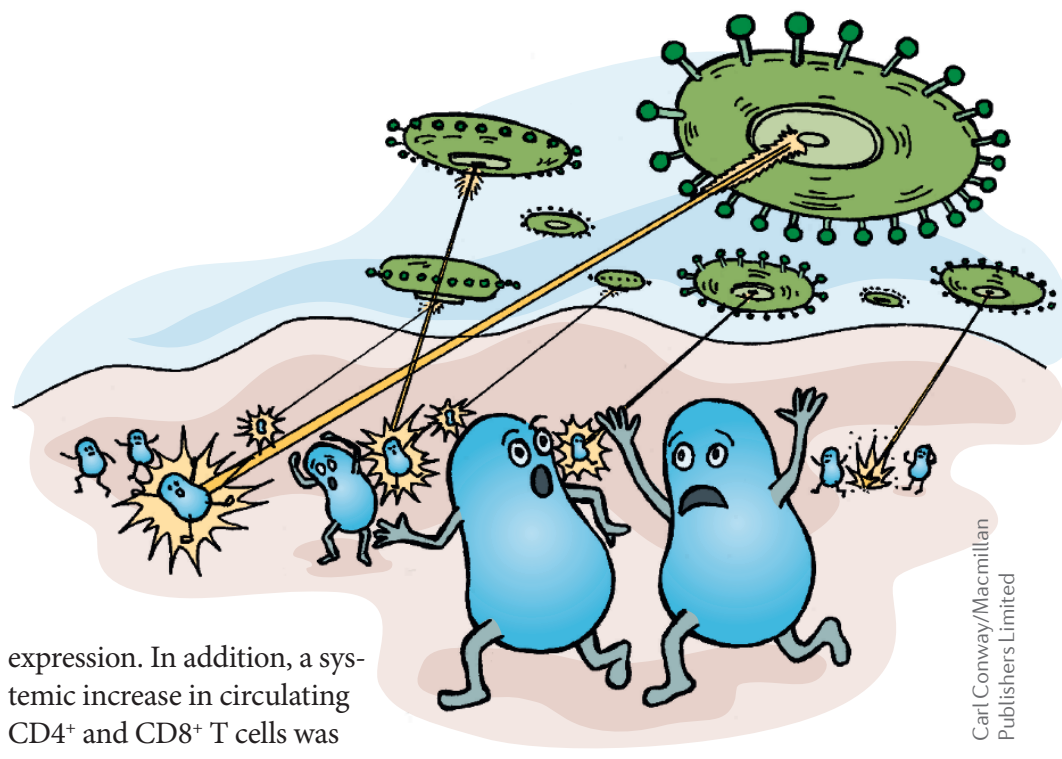

detected. Importantly, the tumour responses were independent of the baseline density of tumour-infiltrating CD8 ${ }^{+} \mathrm{T}$ cells in contrast to responses in patients treated with single-agent anti-PD1 therapies.

Brown et al. reasoned that viral infection of cancer cells would stimulate immune responses equivalent to those elicited by natural infection, whereby an initial innate immune activation could evolve into effective adaptive immunity. To explore this idea, the authors looked at the mechanistic basis of cancer immunotherapy using a hybrid of an oncolytic, non-pathogenic poliovirus (PV) and a human rhinovirus (PVSRIPO). This hybrid was designed to specifically target malignant cells and antigen-presenting cells (APCs), both of which ubiquitously express the PV receptor CD155, and to be incapable of replicating in neurons, to prevent complications such as encephalomyelitis or poliomyelitis. Importantly, PVSRIPO also lacks the capacity to evade or suppress the immune system.

Infection of several cancer cell lines, including melanoma, prostate cancer cells and breast cancer cells, with PVSRIPO resulted in cell lysis with a concomitant release of damage-associated molecular patterns (DAMPs), pattern-associated molecular patterns (PAMPs) and tumour antigens. Furthermore, these DAMPs and PAMPs were sufficient to activate dendritic cells and induce a sustained type I IFN response. In vitro co-culture of immune cells with cancer cell lines revealed that PVSRIPO can also infect APCs in a sublethal manner, leading to co-stimulatory molecule expression, antigen processing, antigen presentation and cytokine production to generate tumour antigen-specific CTL immunity.

As PVs cannot infect mouse cells, demonstrating that this mechanism occurs in vivo required the authors to use a humanized CD155 transgenic mouse model and a modified version of the virus that was capable of replicating in mouse cells (mRIPO). Melanoma cells expressing human CD155 were subcutaneously implanted into immunocompetent mice and treated with a single or mRIPO. The immunotherapy inhibited tumour growth and increased survival of the mice, and these effects were mediated by a combination of direct lysis of cancer cells and pro-inflammatory activation of APCs generating antitumour $\mathrm{T}$ cell responses.

Together, these two studies expand our understanding of the immunotherapeutic potential of oncolytic viruses and suggest that their application in the clinic could enhance the effectiveness of other immunotherapies.

Anna Dart

ORIGINAL ARTICLES Brown, M. C. et al. Cancer immunotherapy with recombinant poliovirus induces IFN-dominant activation of dendritic cells and tumor antigen-specific CTLs. Sci. Transl Med. 9, eaan4220 (2017)|Ribas, A. et al. Oncolytic virotherapy promotes intratumoral $\mathrm{T}$ cell infiltration and improves anti-PD-1 immunotherapy. Cell 170, 1109-1119 (2017) intra-tumoural injection of a control 